\title{
The genetics of migration on the move
}

\author{
Miriam Liedvogel, Susanne Åkesson and Staffan Bensch
}

\author{
CAnMove Centre, Department of Biology, Lund University, Ecology Building, 22362 Lund, Sweden
}

\begin{abstract}
Across a range of organisms, related species or even populations of the same species exhibit strikingly different scales and patterns of movement. A significant proportion of the phenotypic variance in migratory traits is genetic, but the genes involved in shaping these phenotypes are still unknown. Although recent achievements in genomics will evolve migratory genetics research from a phenotypic to a molecular approach, fully sequenced and annotated genomes of migratory species are still lacking. Consequently, many of the genes involved in migration are unavailable as candidates. Migration is central to the life-history adaptations of many animals. Here, we review current understanding of the genetic architecture of migratory traits and discuss the significant implications this will have for other areas of biology, including population responses to climate change, speciation and conservation management.
\end{abstract}

\section{Migration and genetics}

Migration (Box 1) is ubiquitous and enables animals to escape from deteriorating environmental conditions to exploit seasonally favourable habitats. This complex phenomenon includes a suite of morphological, sensory and physiologically adaptive traits that are inherited as components of a 'migratory gene package' (see Glossary; [1,2]), sometimes also discussed in terms of a syndrome (e.g. [3,4]).

Quantitative genetic analyses enable the assessment of heritabilities. Studies of migratory traits have revealed considerable additive genetic variation for single traits (e.g. [5]). Because migration includes a suite of traits, it is also necessary to know the genetic covariation between traits (owing to pleiotropy or linkage) to understand how selection will shape the evolutionary trajectories, as has been shown for other co-adapted traits (reviewed in [6]). Hence, selection of the migratory phenotype will ultimately cause indirect selection on correlated traits and potentially also on traits that have consequences beyond the migratory episode. For example, timing of migration might affect timing of reproduction, emphasising that the field of migration genetics can contribute to the understanding of other life-history events throughout the annual cycle (e.g. breeding biology or physiological adaptations, such as body fat deposition and moult). To achieve insights into the genetic architecture of movement or to understand to what extent the migratory gene package shares similarities between species at different phylogenetic scales, the demonstration of the presence of overall genetic variation is not

Corresponding author: Liedvogel, M. (miriam.liedvogel@biol.lu.se). enough. For such comparative analyses, the genes behind the different components need to be identified.

Given the ground-breaking technological achievements in genomics, it might now be possible to address these questions in migratory non-model species. Here, we outline how insight into the genetic architecture of migration (i.e. the genetic characterisation of migratory phenotypes) will

\section{Glossary}

Candidate gene approach: candidate genes influence the expression of one focal phenotype in one or several (model) organisms and are thus potentially influencing a similar phenotype in other organisms. Candidate trait loci are evaluated based on sequence differences (SNPs, insertion or deletion) between individuals with different phenotypes.

Emlen funnel: orientation cages named after their inventors [65]; they are the traditional tool for analysing orientation behaviour in birds. Using this general principle, migratory direction can be quantified in many ways, both analogue or digitally. The funnel-shaped cage is usually covered with soft pigment paper, enabling scratch marks to be recorded and, therefore, the activity level and migratory direction of the bird to be quantified.

Genome-wide association scans (GWAS): make use of next-generation sequencing technology. This approach enables the entire genome of a large number of phenotypically characterised individuals to be screened to establish associations between genotype and phenotype.

Heritability: the proportion of phenotypic variation within populations explained by additive genetic variation.

Hybrid zone: a contact zone between populations with distinct (migratory) phenotypes. Hybrid zones (and migratory divides) are well-suited scenarios to study processes involved in adaptive divergence and reproductive isolation along ecological gradients.

Linkage disequilibrium: non-random association between alleles at different loci, which might be linked or unlinked.

Migratory divide: the boundary between two adjacent migratory populations with different migratory direction. The divide can be marked by a physical feature, such as a mountain range.

Migratory gene package: in addition to directional information, the gene package also includes genes controlling physiological adaptations necessary for a successful migratory journey, such as timing of migration, body fat deposition and moult $[2,72-74]$. The length of the migratory route appears to be encoded by the circannual clock of the bird [26].

Migratory restlessness ('Zugunruhe'): characteristic behaviour of caged migratory passerines during the migratory period [75]. The birds sit on the perch, flap their wings and try to fly or jump into the side of the cage, pointing in the direction that corresponds to the migratory direction chosen by free-flying conspecifics. In night-migratory birds, the timing and amount of migratory restlessness activity can be easily distinguished from other behaviours, as it occurs only during the migratory period [58].

RNAi: a molecular technique used to decrease the expression of a target gene by disabling the transcribed mRNA via RNA-mediated interference of small fragments of double-stranded RNA whose sequence matches the transcribed sequence of a gene.

RNAseq: highly accurate and efficient approach for transcriptome profiling and characterisation using next-generation sequencing technology.

Selection experiments: experiments in which individuals are selectively mated according to their phenotype (here: migratory status). That is, migrants are mated with migrants and non-migrants with non-migrants for several generations (e.g. [9]). In cross-breeding experiments, migrants are selectively mated with non-migrants, providing some insight into the mode of inheritance of distinct traits (i.e. migratory direction and propensity to migrate).

Zinc-finger nucleases: a class of artificial DNA binding proteins that allow targeted genome manipulation (i.e. gene deletion or insertion) at specified locations via double-strand breaks in the DNA sequence. 
Movement patterns vary in distance directionality and function. Given this complexity, it is essential to define carefully what we mean here by 'movement', and distinguish between different mechanisms, patterns and processes to understand the cause and effect of each movement phenotype (based on $[4,11]$ ).

\section{Dispersal}

Dispersal is defined as the spreading of individuals away from each other (permanently, not including a return). Clobert et al. [11] distinguish between natal dispersal (i.e. offspring movement away from their parents and breeding site), and breeding dispersal (i.e. movement between successive breeding sites). Dispersal determines the range over which genetic mixing occurs and, thus, the degree of homogeneity and inbreeding in one population. From an individual point of view, dispersal is a movement from birth to breeding sites (or between different breeding sites), and its direction can rarely be predicted. Animal dispersal can further involve active exploring of different locations before choosing the most favoured one.

\section{Migration}

Migration is defined as the directional movement of individuals of one species between distinct locations. The timescale on which these movement cycles occur can span hours, days, months or years, or it can be multigenerational. Migration requires a spatiotemporal orientation programme, enabling the animal to return to the same area on a regular (mostly annual) basis.

\section{Station keeping (sedentarism)}

Here, 'sedentarism' is defined as the non-migratory phenotype. During the non-migratory period, the home range or 'centre of activity' of the animal can include daily movement (e.g. foraging and roosting trips, or mate attraction).

Dispersal and migration are similar in that both of them are movement behaviours, and the subset of traits comprising these complex behaviours might overlap to a certain degree (i.e. morphological and physiological adaptations to move per se). However, the selection forces shaping both phenomena appear to be quite different: selection pressures shaping the dispersal phenotype are more likely to be driven by social aspects, whereas resource competition might be more important for migration. For example, if distances covered by a short-distance migrant and by a dispersing animal are similar in dimension, the breeding area for the disperser is determined exactly by the phenotype, whereas the short-distance migrant can have lower site fidelity and, thus, the exact breeding location is not necessarily a consequence of the migratory phenotype. We therefore believe that the selective pressures shaping the phenotype should be different, although dispersal patterns in migratory species might be influenced by their migration routes (perhaps owing to the partly overlapping traits involved in both complexes) [76]. improve understanding of not only the process itself, but also its evolutionary potential.

\section{When is movement migration?}

Migration is a characteristic movement behaviour that not only follows specific routes and timing schedules, but also requires remarkable navigation abilities [2]. Based on quantitative genetics analyses in several insect, fish and bird species, it is well established that a significant proportion of phenotypic variance in migratory traits has a genetic basis. Cross-breeding and selection studies on blackcaps Sylvia atricapilla (Figure 1cii) have further shown that migratory traits are under strong selection and can change within a few generations [7-10]. However, migration is only one of many types of movement (Box 1); therefore, we also discuss its relation to the genetics of other movement phenotypes, particularly dispersal.

\section{Genetics of dispersal}

Dispersal is reported mainly as being condition dependent with no or low heritability; however, despite its importance, it remains a poorly understood behaviour. Dispersal determines the range over which genetic mixing occurs and, therefore, the degree of homogeneity and inbreeding in a population. The decision to disperse is often triggered by environmental and social (rather than genetic) factors and the dispersal phenotype might be strongly shaped by environmental influences during early stages of development (reviewed in [11]). In contrast to primarily environmental and social triggers, several field and selection studies of wing-dimorphic insects [12], plus field studies in some birds [13,14], report high heritabilities of dispersal traits. The ongoing controversy about the presence or absence of a (general) heritable component of dispersal traits is probably at least partly to the result of logistical and methodological challenges; in some studies, both the temporal and spatial scales of the experimental design might have been insufficient to enable a heritable component to be detected [14].

Natal dispersal distance appears to be linked with exploratory behaviour, which has been shown to have a genetic basis in great tits Parus major [15]. Furthermore, an association between exploratory behaviour and a polymorphism in the gene encoding dopamine receptor D4 (DRD4, GenBank accession numbers: DQ006801.1, DQ006802.1) has been found for at least one great tit population $[16,17]$, suggesting a candidate gene for variation in animal movements at a basic level. Similarly, an association between the gene encoding phosphoglucose isomerase (Pgi) and variation in dispersal and flight endurance in Glanville fritillary butterflies Melitaea cinxia, a model species in studies of metapopulation ecology (e.g. $[18,19])$, has been reported [20]. To what extent migration might be governed by similar genes associated with movement at shorter distances, is still an open question and the only test so far was negative. The $D R D 4$ gene was recently tested in blackcaps (HM196845) , 'the' model species in bird migration genetics (Box 2, Figure 1cii,v,vi), but no association with either migratory distance or restlessness behaviour was found [21].

\section{From phenotypic to molecular approaches}

To understand the apparent 'package' response of migratory traits, it is necessary to establish which genes are responsible for the variable expression of specific traits. Three fundamental and challenging questions are: (i) how many genes are involved in modulating migratory strategies? (ii) What is the relative role of structural proteincoding genes and regulatory elements? And, (iii) (how) does the expression of these genes interact with environmental factors? In the context of migration genomics, these questions can best be addressed in species exhibiting a variety of migratory phenotypes, such as the blackcap. This species thus provides an ideal opportunity to study 

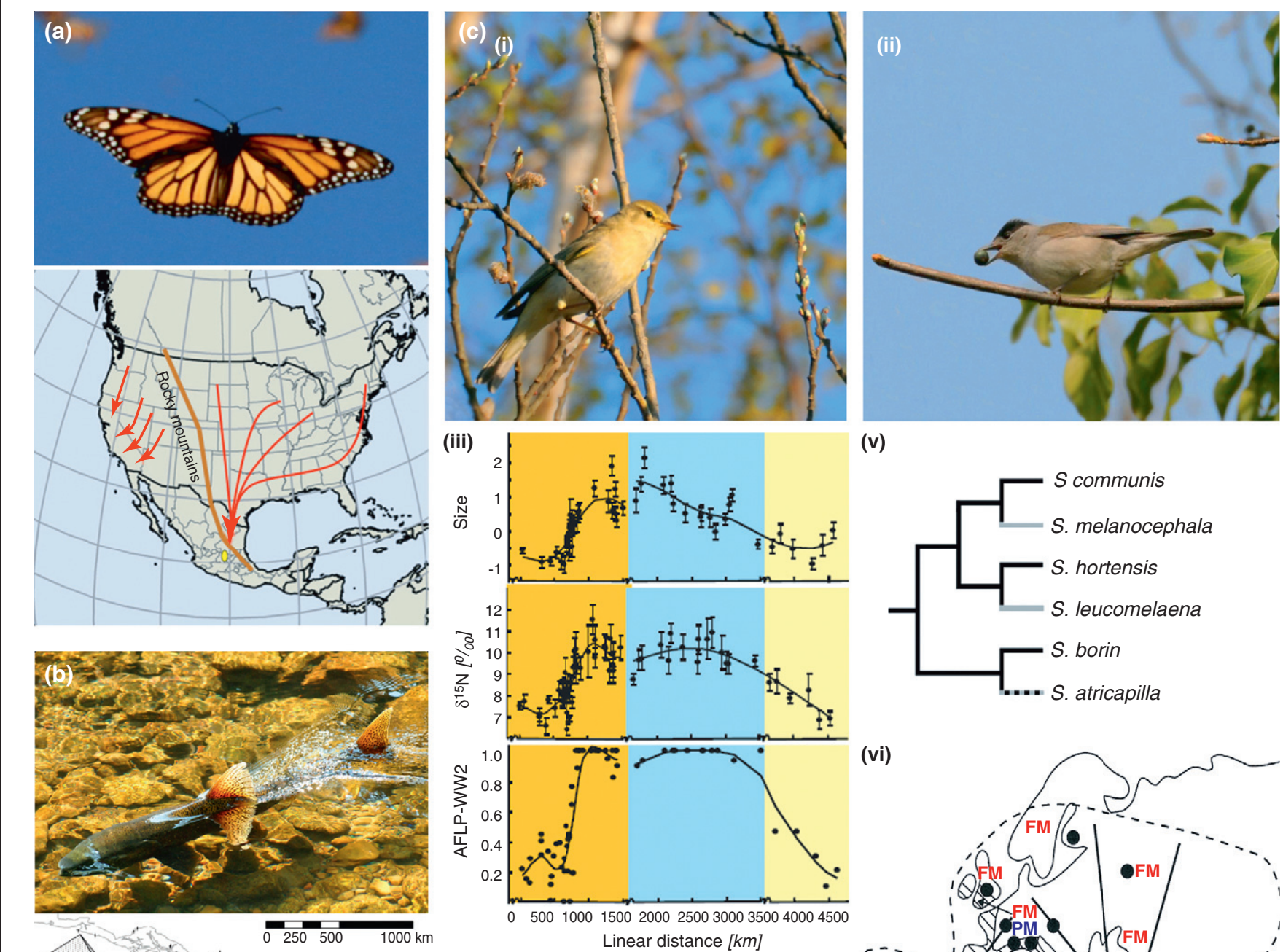

(v)

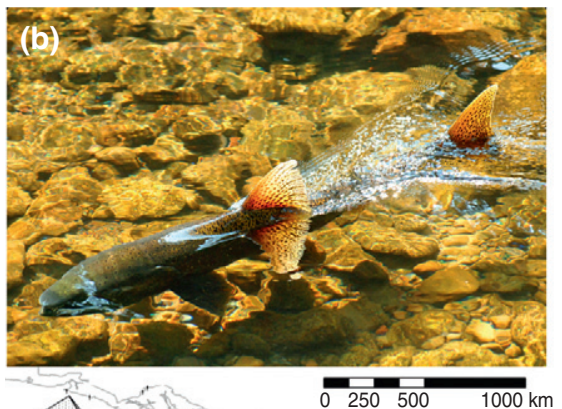

(iv)
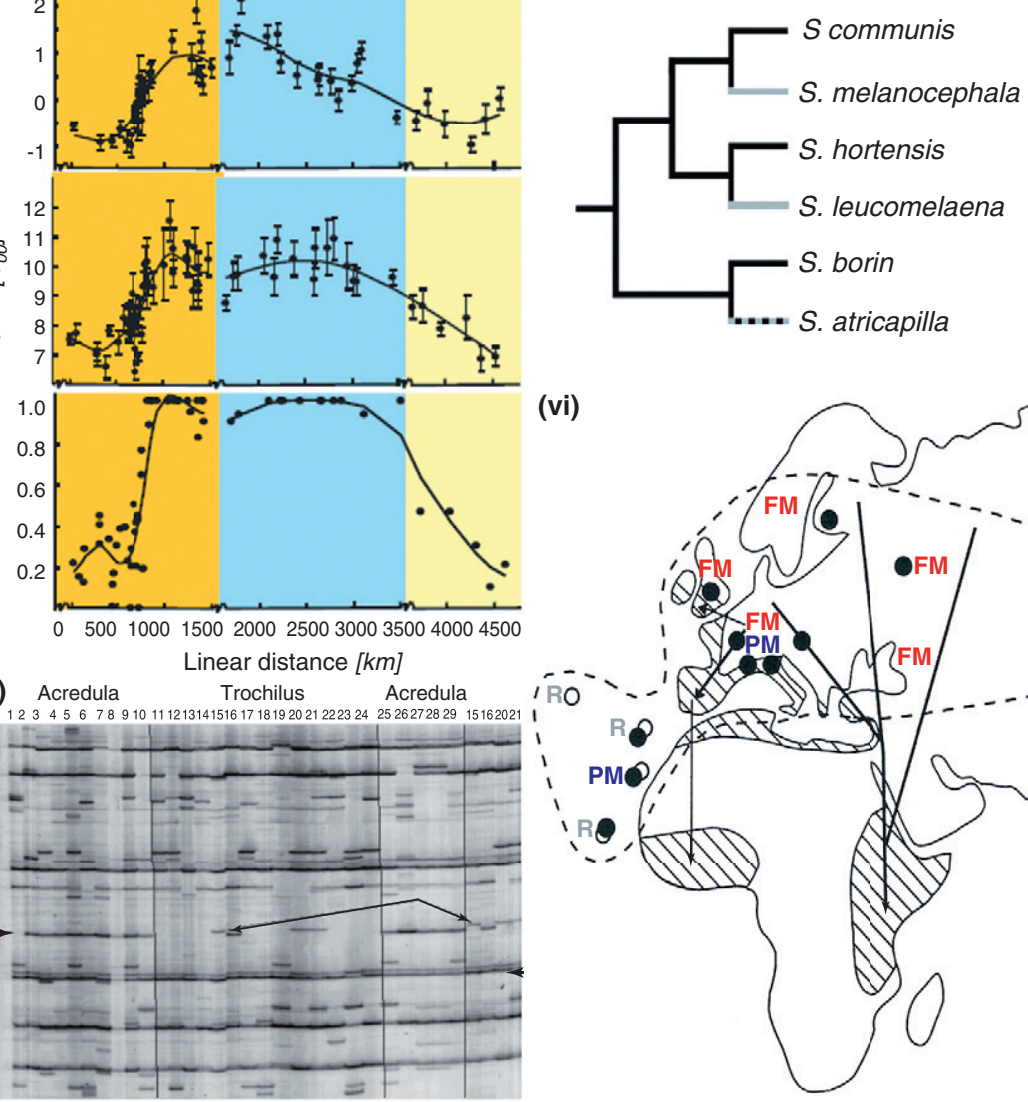

(vi)



TRENDS in Ecology \& Evolution

Figure 1. Useful model organisms with which to study the genetics of migration in different taxa. (a) Monarch butterfly Danaus plexippus. Eastern North American monarch butterflies migrate south during the fall migration (red arrows east of the Rocky Mountains). Their long-distance migratory route leads to a focal destination in central Mexico (yellow oval). The monarch butterfly population west of the Rocky Mountains undergoes a shorter fall migration, overwintering in roosts along the Pacific Coast (red arrows west of the Rocky Mountains). Because of the distances travelled and the nature of the focal stopping point, most navigational and genetic research focuses on the eastern North American migration. (b) Chinook salmon Oncorhynchus tshawytscha. Variation in run timing of Chinook salmon populations across a transect from the southern end of the range of the species north to southeast Alaska. (c) Two migratory birds with populations exhibiting various migratory strategies: (i) European blackcap Sylvia atricapilla and (ii) willow warbler Phylloscopus trochilus. (iii) Variation in phenotypic traits (body size and stable isotope $\delta^{15} \mathrm{~N}$ values), and allele frequency for amplified fragment length polymorphism (AFLP)-WW2, a genetic marker for migratory direction in the willow warbler, around the Baltic along three segments: southern Sweden to northwestern Norway (orange); northwestern Norway to Lithuania (blue) and eastern Poland to Denmark (grey). (iv) AFLP profile of two willow warbler subspecies (P.t. acredula and P.t. trochilus); (v) Variation in migratory behaviour between six closely related species of Sylvia warblers. Unbroken black branches indicate long-distance migrants, unbroken grey branches indicate resident species; the broken grey branch mirrors the highly variable migratory phenotype in blackcaps. (vi) Schematic representation of within-species variation of migratory strategies and routes in blackcaps. Key: FM, fully migratory; PM, partially migratory; R, residents; main migratory routes are represented as arrows; broken lines show outer limits of the breeding range, hatched areas mark the wintering grounds. Reproduced, with permission, from [52] (a), Lynn Ketchum (b; photo); Gernot Segelbacher (ci, ii); and [34] (civ); modified with permission from [77] (b); [38] (ciii); [3] (cv) and [1] (cvi). 
To apply the toolset of genomics successfully to any particular question in migration genetics research, it is key to select carefully both the most suitable study species and the optimal experimental design.

\section{Phenotype quantification}

For all studies on the genetics of migration, it is crucial to characterise, quantify and control the focal migratory trait accurately (i.e. propensity to migrate, direction or timing parameters). To study the propensity to migrate, species such as blackcaps with different populations exhibiting a broad repertoire of migratory strategies (i.e. sedentary and migratory populations) are useful models (Figure 1cvi). Furthermore, in blackcaps, migratory restlessness activity can be used as a proxy for the migratory activity per se. Timing (onset and duration) and amount of migratory restlessness movement is easy to quantify under controlled conditions. Migratory direction in small passerines is more difficult to quantify with the necessary accuracy on an individual level, but future advances in tracking technology are likely to provide the opportunity to track the migratory route of migratory passerines more precisely.

\section{Comparative genomics}

For comparative genetics and/or genomics approaches, it is essential that closely related species or different populations of the model species express a diversity of migration patterns. Ideal model groups of species for this purpose are those with complex phylogenetic patterns in the presence and absence of migratory behaviour (i.e. populations exhibiting diverse spectra of migratory strategies).

Pacific Northwest salmon are a suitable model genus to study spatiotemporal aspects of migration. The economic value and, thus, the interest in monitoring programmes provide an additional pool of excellent phenotypic information (namely, the availability of advanced tracking data characterising intra- and interspecies spatial orientation and temporal return patterns within this genus) to study movement patterns and overall spatial distribution.
Furthermore, many bird groups (e.g. Sylvia, Phylloscopus and Dendroica warblers, and wheatears Oenanthe spp. or Catharus spp.) with a broad range of migratory phenotypes are useful models to study similarities and differences between closely related species at different phylogenetic scales using a comparative genomics approach (Figure 1, main text).

\section{Genotype-phenotype association}

To test for the crucial effect of a candidate gene in the focal migratory phenotype expression, it is necessary to modify the genotype (i.e. knockout and rescue mutants). Insects are ideal for this purpose; they have short generation times and are routinely bred in the laboratory in large numbers. One useful model system is the monarch butterfly. The migratory cycle in monarch butterflies is multigenerational: all generations are equipped with the same core genetic material, and have the prerequisites to migrate. Drosophila as an insect model species can be used to genetically modify and test the phenotypic effect of at least some (not monarch specific) candidate genes. Furthermore, zinc-finger nucleases provide one promising tool for knocking out candidate migration genes in the monarch to show their function in shaping the migratory phenotype. The use of RNAimediated post-transcriptional gene silencing is also a possibly useful technology.

\section{Gene mapping}

Traditionally, gene mapping requires pedigree information from wild populations or captive populations selectively bred to generate a $F_{2}$ or backcross population, in combination with genetic markers on a linkage map. Constructing sufficiently large pedigrees or crossbred captive populations (several hundred to thousand individuals) is both labour and cost intensive. Genome-wide association scans (GWAS) are an alternative approach offered by next-generation sequencing technology that can be used in open populations but typically requires thousands of individuals.

Figure 1), is likely to revolutionise the field of migratory genetics and establish a new field of research, that of migratory genomics.

As an alternative to comparing genotypes of individuals exhibiting different migratory traits, one can make use of the fact that, in migratory species, the migratory phenotype is only expressed during certain time periods. This suggests that (at least for birds), once it is possible to characterise the expression profiles in a blood sample (e.g. via microarray or RNAseq technologies), researchers will be able to map (and ultimately manipulate) expression differences within the same individual over time, which will keep 'background noise' to a minimum.

Comparative analyses of gene expression profiles of sedentary and migratory individuals of the same population (i.e. individuals that are very similar in terms of their genes but differ in terms of their phenotypes) are an ideal way of identifying genes and networks of genes within the migratory gene package $[40,41]$. This approach is based on mRNA extracts from different tissues (e.g. brain, eye or liver) and uses microarray analysis or transcriptome sequencing to identify genes that are differentially regulated between different phenotypic groups. However, the genetic variation in migratory traits within and between populations (as demonstrated by quantitative genetics) might not necessarily be found in the sequences of those differentially regulated genes, but rather in regulatory elements upstream or elsewhere in the genome [42]. Here, we outline state-of-the-art research currently in progress and discuss key studies in insects, fishes and birds (Figure 1, Box 2). 


\section{Should I stay or should I go? Genes regulating the propensity to migrate}

Most of the current understanding of the genetic basis and evolutionary aspects of bird migration and migratory traits is based on classic crossbreeding and selection experiments on blackcaps (Figure 1c). These results clearly showed that there is considerable genetic variation in migratory traits and that they are 'linked' (or genetically correlated) and inherited as components of a migratory gene package [1], indicating that selection can change suites of traits and that certain changes can occur very rapidly $[5,10,43]$. The results also suggest that many, if not all, birds have the genetic machinery to migrate and, with appropriate selection pressure, migratory or sedentary behaviour can dominate within a population. A switch to the alternative behaviour can occur within a few generations, particularly in populations that include a continuum of migratory strategies, thus demonstrating substantial additive genetic variation for migratory traits [9,10,44-48]. Yet, how a complex multi-gene adaption such as migration can repeatedly be lost and gained in the course of evolution cannot be understood until its genetic architecture has been disentangled.

A recent study in blackcaps investigated polymorphisms in six previously identified candidate genes for behavioural traits that might be linked to migration. Allelic variation at one candidate locus, the ADCYAP1 gene, significantly correlated with migratory restlessness (a proxy for migratory activity in night-migratory songbirds); longer alleles were correlated with higher migratory activity [21]. This association was found both within and across populations, which strengthens the result. However, linkage disequilibrium can extend for several $100 \mathrm{~kb}$ (or even $\mathrm{Mb}$ [49]); thus, further investigation of additional markers in the chromosomal region is needed to confirm whether the actual gene involved is $A D C Y A P 1$. To test the generality of this finding, future studies are needed to determine whether this locus is also associated with migratory traits in other passerines.

The migratory cycle of the eastern North American monarch butterflies Danaus plexippus is exceptional in the way that it involves successive generations (reviewed in [50]; Figure 1a). Those individuals migrating south every fall are thus on their maiden voyage and so migration must have a genetic basis. Because all butterflies have the same core genetic material and all generations can migrate when exposed to the relevant stimuli (which are still not completely understood), it is possible that epigenetic mechanisms are key to triggering the changes in gene expression or function that underlie the migratory phenotype. More precisely, there is a genetic basis to the entire migratory process, but the generational differences in phenotype might be epigenetically regulated. The toolbox available for the monarch butterfly contains a monarch cell line and an EST library (from brain tissue) for transcriptional comparison between migratory and summer monarch butterflies; the fully sequenced and annotated monarch genome will also soon be available (e.g. [41,51]; reviewed in [52]). Gene expression analyses of migratory and nonmigratory monarch butterflies have identified 40 differentially expressed genes ( $>60 \%$ of which are annotated [41]).
As for many insect species, another advantage of studying monarch butterflies is the possible use of Drosophila as a model for genetic manipulations of the genotype. Ultimately, the Drosophila model will enable the effect of some candidate monarch genes on phenotype expression and regulation to be tested (e.g. magnetosensitive proteins [52]). The use of zinc-finger nucleases is a promising strategy for targeting candidate genes, not only with respect to knockout, but also for introducing reporter tags into target loci within the monarch genome [52]. The authors promote the transfer of this method to not only other non-model insects, but also non-model vertebrates. This is a promising perspective, although the applicability in other study organisms remains to be tested.

\section{Where do we go? Genes determining direction of migration}

Highly synchronised and directional movement of animals is a key trait of the migratory phenotype. This is in contrast to dispersal, where individuals are spreading away from others, with the direction of each individual being rarely predictable. Current understanding of the genetic basis of directional information in birds is based on two types of experiment: crossbreeding experiments (e.g. $[9,53])$ and displacement experiments (e.g. [54,55]). Crossbreeding experiments of blackcaps suggest that migratory direction is inherited as one component of a migratory gene package [1]. Genetically determined migratory direction is susceptible to small evolutionary changes that can be detected on a population level (i.e. microevolution) [9]. A recent study on blackcaps further suggests associated morphological changes (i.e. wing and beak shape) in response to a newly established migratory route [48].

Not only birds, but also many fish species perform spectacular seasonal mass migrations. As these mass movements occur below the ocean surface, most of the details remain unknown. There is some (indirect) evidence for a genetic basis of migratory direction from a study on Atlantic eels. Migratory journeys of American Anguilla rostrata and European eel Anguilla anguilla start in the Sargasso Sea, but their migratory routes differ in distance and direction. America eels migrate towards the North American coast and European eels follow a slightly longer northeasterly route toward Europe. By contrast, Icelandic eels follow an intermediate migratory direction. A recent study using AFLP as a method to characterise hybrids, identified a high proportion of Icelandic eels as hybrids, suggesting an intermediate mode of inheritance of migratory direction [37].

Another avian model organism for migratory research is the willow warbler Phylloscopus trochilus (Figure 1ci). As in blackcaps and other species of birds [56], two populations of willow warbler with different migratory directions meet and form a so-called 'migratory divide' [33-35]. Despite strong differences in migratory behaviours and partly in morphology, there is no apparent genetic difference in neutral markers across the willow warbler migratory divide [38]. However, two AFLP markers derived from a whole-genome scan, show significant variation across the migratory divide in Scandinavia [34], and the allele frequency distribution of one of the identified AFLP markers 
also matches the more gradual change of migratory direction in Poland [38] (Figure 1ciii,iv). Subsequent characterisation and mapping of the chromosomal location of this marker on the willow warbler genome will enable the gene(s) under selection to be identified.

\section{When? Genes timing migration}

In migratory birds, the length of the migratory route appears to be encoded by the circannual clock of the bird, which senses changes in photoperiod (day length) and mediates seasonal behaviours. The period during which caged migrants show migratory restlessness behaviour is in accordance with onset, duration and termination of actual migration in wild conspecifics (e.g. $[57,58]$ ). Thus, migratory restlessness is a good (i.e. easy to quantify) proxy for migratory activity. The inherited time programme equips the birds with information on when to leave the breeding area, when to stop, and how fast and in which direction to fly $[2,26]$.

One driving force in recent studies on fish migration has been the economic value of some species (e.g. many juveniles are tagged with coded wire tags when released from hatcheries, enabling their movement pattern and overall spatial distribution to be tracked). One model well suited to the study of the spatiotemporal aspects of fish migration genetics is the Pacific Northwest salmon (Figure 1b) (e.g. [59]). The genus Oncorhynchus comprises seven different species showing high variability in both spatial orientation and temporal return patterns within and among species. How can genomics help to understand the spatiotemporal aspects of pacific salmon migration? As in other study species, the strong phenotypic pattern found in Pacific Northwest salmon is not reflected in overall genetic differentiation [36]. However, a candidate gene approach led to the discovery of a latitudinal cline in Clock, a central and well molecularly characterised component of the circadian clock in animals $[28,29]$. The identified Clock gene (OtsClock1b, DQ780895) variation corresponds to the cline in spawning time in the Chinook salmon Oncorhynchus tshawytscha, which is earlier in the north, and demonstrated that day length is a primary cue for migrating and spawning in these fish $[28,29]$. These findings suggest that Clock is involved in mediating seasonal adaptation and influences geographical variation in reproductive timing in at least some migratory salmon species. An earlier study on blue tits Cyanistes caeruleus also reported a latitudinal cline in Clock gene (AY376853) variability, which might reflect a general adaptation to ecological factors correlated with latitude [60]. This finding also highlights the potential of how understanding aspects of the genetic architecture of migratory traits can be integrated with, and complement research on, other lifehistory traits (e.g. timing of breeding biology). Furthermore, a possible consequence of a connection between timing of migration and breeding is reproductive isolation between sympatric populations [61]. In this context, it has been suggested that migration is a 'magic trait' as selection (directly or indirectly) on migratory timing alone might result in allochrony, reproductive isolation and eventually speciation $[62,63]$. Hence, the field of genetics of migration might provide important insights into the field of genetics of speciation.

\section{Challenges and future perspectives}

Studying the genetics of migration is challenging because migratory behaviours are complex and difficult to clearly define and manipulate; in addition, migratory species are non-model organisms for which genetic tools are lacking. To identify genes and understand the genetic architecture underlying the variability of migratory phenotypes to draw evolutionary conclusions, it is crucial to: (i) clearly define, describe, quantify and distinguish the traits of the focal phenotype (i.e. direction, migratory restlessness activity or timing parameters); (ii) disentangle genetic and plastic effects underlying migration; and ultimately (iii) understand fitness consequences associated with the migratory phenotype. We believe that a highly integrative approach to study the genetics of migration is imperative [64]. Room for interaction, exchange of ideas and critical assessment of methodological approaches and their potential is essential, particularly in light of the newly emerging research direction of migratory genetics. One constructive solution to provide the opportunity for this dialogue is via small meetings, workshops and symposia.

We are convinced that, owing to technical developments and rapid progress in technology, the genetics of migration field is already evolving into one of migration genomics. Thus, genomic resources, such as EST-based microarray analyses, transcriptome libraries and single nucleotide polymorphism (SNP) marker sets will soon be (or have already become) available for migratory species (Box 2, Figure 1). We also emphasise that it is necessary to focus on improving and developing accurate methods for scoring and manipulating the migratory phenotype. Unless clearly defined and accurately quantified traits are available, full genome sequences or tissue-specific expression analyses are unlikely to result in many insights into understanding the underlying mechanisms or evolutionary potential. Hence, the field of migratory genetics must move along both fronts in parallel, combining the resources offered by next-generation sequencing with cutting-edge technology to quantify the migratory phenotype.

In addition, all fully annotated genomes that have been used as references for transcriptome and/or genome annotation of migratory species so far come from non-migratory animals (e.g. Drosophila and zebra finch Taeniopygia guttata). Hence, it is unclear whether the genes being looked for are expressed, functional or even occur in the model organisms used as default references. Thus, the genomics of migration with sequenced and in the future also (fully) annotated genomes for migratory species will enable researchers to validate the function of the identified gene candidates.

Quantification of morphological traits is generally straight forward, but accurate quantification of complex behavioural traits, such as migratory activity and direction, is more challenging. The traditional tool for analysing orientation behaviour in birds is the so-called 'Emlen funnel' [65]. This method enables general differences in migratory direction between experimental groups to be assessed, but one can doubt whether this level of accuracy will be sufficient for precise gene mapping. There is also significant progress being made in the development of miniaturised tracking devices [66], and precise tracking 
data of the complete migratory path will gradually become available also for smaller sized migrants.

Currently, much effort is being put into investigating the movement patterns of many different species. To make costly satellite tracking data accessible to studies of migration genetics, which require sample sizes of several hundred individuals, synchronised activities are required that focus on several populations of one model species. This would enable researchers to integrate these data with whole-genome association studies. A candidate model species for birds is the osprey Pandion haliaetus, a nonflocking migrant with a worldwide distribution with various migration patterns that has already been demonstrated to be a good target for satellite tracking (e.g. [67]). Given that ospreys travel solitarily and fledge more than one offspring, researchers can make use of full-sib analyses, preferably after cross-fostering. This would enable genes to be associated to different components of the migratory package as retrieved from the tracked migratory path. In addition, ospreys show high breeding site fidelity; thus, individuals can be recaptured when necessary (e.g. for battery replacement). The dimension of such a project is beyond the scope of what a single research group can cover and therefore calls for broad collaborative efforts. As tracking techniques advance, the number of potential candidate species will increase, leading to the possibility of such large-scale projects on passerine birds (e.g. blackcaps or flycatchers). The massive data set that will be created by joint efforts will not only be unique in its benefit for migration genetics, but will also further provide otherwise inaccessibly high resolution comparative phenotype, genetic and environmental data for other areas of biology (e.g. breeding biology or conservation management). Tracking the route and timing of migration of single individuals and their offspring from several populations over multiple seasons in combination with genome-wide association scans [39] (Box 2), will ultimately allow researchers to distinguish whether an observed change in migration is a phenotypically plastic response to climate change or an evolutionary response or possibly a combination of both [68]. This multi-population approach will further allow researchers to disentangle how and under which circumstances timing of migration is constrained by environmental variables.

Conservation and habitat management strategies will benefit significantly from a basic understanding of the genetics of migration. Once genetic components of migration are identified (e.g. genes determining the timing of migration), it will be possible to predict which migratory species might be especially vulnerable to climate change. This will further enable the assessment of the relative contributions of plastic and genetic response patterns of migratory species to global warming, which might differ both between and within species.

Focusing on both the impact and magnitude of the effect of environmental variables will be key to understanding the putative regulatory network of environment and genotype interactions shaping the focal phenotype. The expression of some migratory traits is regulated by external cues, and differences might also be epigenetic or plastic responses. Changes in day length can trigger onset of migratory restlessness [69], and alterations of the magnetic field have been shown to modulate fuel deposition in birds [70]. The most dramatic example is probably the transition to the migratory phenotype in the desert locust Locusta migratoria, which is triggered by increased serotonin levels owing to social interactions [71]. The ideal experiment to disentangle genetic and plastic effects would be to carry out common garden experiments; although this is a challenging, labour and cost-intense approach, it is one that could be realised with synchronised efforts, as outlined above.

\section{Concluding remarks}

We believe that transcriptome characterisation in combination with gene mapping studies and gene expression profiling will provide the raw material to tackle key questions in the field of migration genetics outlined and discussed here. Once differently expressed gene or variable genomic region candidates have been identified, it will be possible to ascertain how this variation relates to the phenotypes of individuals, and how epigenetic changes contribute to the variation in migratory traits. Consequently, this will lead to an understanding of the genetic architecture of complex traits and, ultimately, the association between variation in the environment and the genotype of an individual.

Given the variability of migratory phenotypes both at the individual and the population level, migration is a useful phenomenon to study genetic architecture as a function of life-history variation. To make major achievements in this field, it will be necessary to combine next-generation sequencing tool sets with cutting edge technology to quantify the migratory phenotype. We also want to stress the importance of not analysing the adaptations in migration and their evolutionary change in isolation, but to integrate and complement these with additional studies on other key life-history adaptation, such as breeding biology, within the annual cycle.

\section{Acknowledgements}

This work was supported by the Centre for Animal Movement Research (CAnMove) financed by a Linnaeus grant (349-2007-8690) from the Swedish Research Council and Lund University; and a Feodor Lynen fellowship, Alexander von Humboldt-Foundation (ML).

\section{References}

1 Berthold, P. (1999) A comprehensive theory for the evolution, control and adaptability of avian migration. Ostrich 70, 1-11

2 Åkesson, S. and Hedenström, A. (2007) How migrants get there: migratory performance and orientation. BioScience 57, 123-133

3 Piersma, T. et al. (2005) Is there a 'migratory syndrome' common to all migrant birds? Ann. N. Y. Acad. Sci. 1046, 282-293

4 Dingle, H. (2006) Animal migration: is there a common migratory syndrome? J. Ornithol. 147, 212-220

5 Pulido, F. and Berthold, P. (2003) Quantitative genetic analysis of migratory behavior. In Avian Migration (Berthold, P. et al., eds), pp. 53-77, Springer

6 Sinervo, B. and Svensson, E. (2002) Correlational selection and the evolution of genomic architecture. Heredity 89, 329-338

7 Berthold, P. (1991) Spatiotemporal programmes and genetics of orientation. In Orientation in Birds (Berthold, P., ed.), pp. 86-105, Basel, Birkhäuser

8 Berthold, P. (1991) Genetic control of migratory behavior in birds. Trends Ecol. Evol. 6, 254-257

9 Berthold, P. et al. (1992) Rapid microevolution of migratory behavior in a wild bird species. Nature $360,668-670$ 
10 Pulido, F. and Berthold, P. (2010) Current selection for lower migratory activity will drive the evolution of residency in a migratory bird population. Proc. Natl. Acad. Sci. U.S.A. 107, 7341-7346

11 Clobert, J. and et, al., eds (2001) Dispersal, Oxford University Press

12 Roff, D.A. and Fairbairn, D.J. (2001) The genetic basis of dispersal and migration and its consequences for the evolution of correlated traits. In Dispersal (Clobert, J. et al., eds), pp. 191-202, Oxford University Press

13 Hansson, B. et al. (2003) Heritability of dispersal in the great reed warbler. Ecol. Lett. 6, 290-294

14 Pasinelli, G. et al. (2004) Genetic and environmental influences on natal dispersal distance in a resident bird species. Am. Nat. 164, 660-669

15 Dingemanse, N.J. et al. (2003) Natal dispersal and personalities in great tits (Parus major). Proc. R. Soc. B 270, 741-747

16 Fidler, A.E. et al. (2007) Drd4 gene polymorphisms are associated with personality variation in a passerine bird. Proc. R. Soc. B 274, 1685-1691

17 Korsten, P. et al. (2010) Association between DRD4 gene polymorphism and personality variation in great tits: a test across four wild populations. Mol. Ecol. 19, 832-843

18 Hanski, I. and Saccheri, I. (2006) Molecular-level variation affects population growth in a butterfly metapopulation. PLoS Biol. 4, e129

19 Vera, J.C. et al. (2008) Rapid transcriptome characterization for a nonmodel organism using 454 pyrosequencing. Mol. Ecol. 17, $1636-1647$

20 Niitepold, K. et al. (2009) Flight metabolic rate and Pgi genotype influence butterfly dispersal rate in the field. Ecology 90, 2223-2232

21 Mueller, J.C. et al. (2011) Identification of a gene associated with avian migratory behaviour. Proc. R. Soc. B 278, 2848-2857

22 Gwinner, E. (1967) Circannuale Periodik der Mauser und der Zugunruhe bei einem Vogel. Naturwissenschaften 54, 447

23 Gwinner, E. (1986) Circannual rhythms in the control of avian migrations. Adv. Stud. Behav. 16, 191-228

24 Gwinner, E. (1996) Circadian and circannual programmes in avian migration. J. Exp. Biol. 199, 39-48

25 Berthold, P. (1984) The endogenous control of bird migration - a survey of experimental evidence. Bird Study 31, 19-27

26 Pulido, F. et al. (2001) Heritability of the timing of autumn migration in a natural bird population. Proc. R. Soc. B 268, 953-959

27 Anderson, J.J. and Beer, W.N. (2009) Oceanic, riverine, and genetic influences on spring chinook salmon migration timing. Ecol. Appl. 19, 1989-2003

28 O'Malley, K.G. and Banks, M.A. (2008) A latitudinal cline in the Chinook salmon Oncorhynchus tshawytscha Clock gene: evidence for selection on PolyQ length variants. Proc. R. Soc. B 275, 2813-2821

29 O'Malley, K.G. et al. (2010) Clock polymorphism in Pacific salmon: evidence for variable selection along a latitudinal gradient. Proc. $R$. Soc. B 277, 3703-3714

30 Froy, O. et al. (2003) Illuminating the circadian clock in monarch butterfly migration. Science 300, 1303-1305

31 Helbig, A.J. (1996) Genetic basis, mode of inheritance and evolutionary changes of migratory directions in Palearctic warblers (Aves: Sylviidae). J. Exp. Biol. 199, 49-55

32 Buerkle, C.A. (1999) The historical pattern of gene flow among migratory and nonmigratory populations of prairie warblers (Aves: Parulinae). Evolution 53, 1915-1924

33 Bensch, S. et al. (1999) Morphological and molecular variation across a migratory divide in willow warblers, Phylloscopus trochilus. Evolution 53, 1925-1935

34 Bensch, S. et al. (2002) The use of AFLP to find an informative SNP: genetic differences across a migratory divide in willow warblers. Mol. Ecol. 11, 473-481

35 Pérez-Tris, J. et al. (2004) Historical diversification of migration patterns in a passerine bird. Evolution 58, 1819-1832

36 O'Malley, K.G. and Banks, M.A. (2007) Candidate loci reveal genetic differentiation between temporally divergent migratory runs of Chinook salmon (Oncorhynchus tshawytscha). Mol. Ecol. 16, $4930-4941$

37 Albert, V. et al. (2006) Natural hybrids in Atlantic eels (Anguilla anguilla A. rostrata): evidence for successful reproduction and fluctuating abundance in space and time. Mol. Ecol. 15, 1903-1916

38 Bensch, S. et al. (2009) Genetic, morphological, and feather isotope variation of migratory willow warblers show gradual divergence in a ring. Mol. Ecol. 18, 3087-3096
39 Stapley, J. et al. (2010) Adaptation genomics: the next generation. Trends Ecol. Evol. 25, 705-712

40 Jones, S. et al. (2008) Changes in brain gene expression during migration in the white-crowned sparrow. Brain Res. Bull. 76, 536-544

$41 \mathrm{Zhu}, \mathrm{H}$. et al. (2009) Defining behavioral and molecular differences between summer and migratory monarch butterflies. BMC Biol. 7, 14

42 Carroll, S.B. (2005) Evolution at two levels: on genes and form. PLoS Biol. 3, e245

43 Pulido, F. and Berthold, P. (1998) The microevolution of migratory behaviour in the blackcap: effects of genetic covariances on evolutionary trajectories. Biol. Conserv. Fauna 102, 206-211

44 Berthold, P. and Helbig, A.J. (1992) The genetics of bird migration: stimulus, timing and direction. Ibis 134 (Suppl.), 35-40

45 Berthold, P. and Pulido, F. (1994) Heritability of migratory activity in a natural bird population. Proc. R. Soc. B 257, 311-315

46 Berthold, P. (ed.) (2000) Vogelzug - Eine aktuelle Gesamtübersicht, Wissenschaftliche Buchgesellschaft

47 Outlaw, D.C. and Voelker, G. (2006) Phylogenetic tests of hypotheses for the evolution of avian migration: a case study using the Motacillidae. Auk 123, 455-466

48 Rolshausen, G. et al. (2009) Contemporary evolution of reproductive isolation and phenotypic divergence in sympatry along a migratory divide. Curr. Biol. 19, 2097-2101

49 Lundberg, M. et al. (2011) Identification of a divergent chromosome region in a non-model species, the willow warbler Phylloscopus trochilus, by a cross-species use of genomic resources. J. Evol. Biol. 24, 1241-1253

50 Brower, L.P. (1996) Monarch butterfly orientation: missing pieces of a magnificent puzzle. J. Exp. Biol. 199, 93-103

$51 \mathrm{Zhu}, \mathrm{H}$. et al. (2008) Chasing migration genes: a brain expressed sequence tag resource for summer and migratory monarch butterflies (Danaus plexippus). PLoS ONE 3, e1345

52 Reppert, S.M. et al. (2010) Navigational mechanisms of migrating monarch butterflies. Trends Neurosci. 33, 399-406

53 Helbig, A.J. (1994) Genetic basis and evolutionary change of migratory directions in a European passerine migrant Sylvia atricapilla. Ostrich $65,151-159$

54 Perdeck, A.C. (1958) Two types of orientation in migrating starlings Sturnus vulgaris $L$. and chaffinches Fringilla coelebs $L$., as revealed by displacement experiments. Ardea 46, 1-37

55 Chernetsov, N. et al. (2008) A long-distance avian migrant compensates for longitudinal displacement during spring migration. Curr. Biol. 18, 188-190

56 Irwin, D.E. and Irwin, J.H. (2005) Siberian migratory divides. The role of seasonal migration in speciation. In Birds of Two Worlds (Greenberg, R. and Marra, P.P., eds), pp. 27-40, Johns Hopkins University Press

57 Berthold, P. (1973) Relationships between migratory restlessness and migratory distance in six Sylvia species. Ibis 115, 594-599

58 Berthold, P. and Querner, U. (1988) Was Zugunruhe wirklich ist - eine quantitative Bestimmung mit Hilfe von Video-Aufnahmen bei Infrarotlichtbeleuchtung. J. Ornithol. 129, 372-375

59 Weitkamp, L.A. (2010) Marine distributions of Chinook salmon from the west coast of North America determined by coded wire tag recoveries. Trans. Am. Fish. Soc. 139, 147-170

60 Johnsen, A. et al. (2007) Avian Clock gene polymorphism: evidence for a latitudinal cline in allele frequencies. Mol. Ecol. 16, 4867-4880

61 Friesen, V.L. et al. (2007) Sympatric speciation by allochrony in a seabird. Proc. Natl. Acad. Sci. U.S.A. 104, 18589-18594

62 Servedio, M.R. et al. (2011) Magic traits in speciation: 'magic' but not rare? Trends Ecol. Evol. 26, 389-397

63 Price, T. (2008) Speciation in Birds, Roberts \& Company Publishers

64 Bowlin, M.S. et al. (2010) Grand challenges in migration biology. Integr. Comp. Biol. 50, 1-19

65 Emlen, S.T. and Emlen, J.T. (1966) A technique for recording migratory orientation of captive birds. Auk 83, 361-367

66 Robinson, W.D. et al. (2010) Integrating concepts and technologies to advance the study of bird migration. Front. Ecol. Environ. 8, 354-361

67 Hake, M. et al. (2001) Satellite tracking of Swedish ospreys Pandion haliaetus: autumn migration routes and orientation. J. Avian Biol. 32 , 47-56

68 Alerstam, T. et al. (2003) Long-distance migration: evolution and determinants. Oikos 103, 247-260 
69 Helm, B. and Gwinner, E. (2006) Migratory restlessness in an equatorial non-migratory bird. PLoS Biol. 4, e110

70 Fransson, T. et al. (2001) Magnetic cues trigger extensive refuelling. Nature 414, 35-36

71 Anstey, M.L. et al. (2009) Serotonin mediates behavioural gregarization underlying swarm formation in desert locusts. Science 323, 627-639

72 Berthold, P. and Querner, U. (1981) Genetic basis of migratory behavior in European warblers. Science 212, 77-79

73 Berthold, P. and Querner, U. (1995) Microevolutionary aspects of bird migration based on experimental results. Israel J. Zool. 41, 377-385
74 Bairlein, F. (2002) How to get fat: nutritional mechanisms of seasonal fat accumulation in migratory songbirds. Naturwissenschaften 89, $1-10$

75 Kramer, G. (1949) Über Richtungstendenzen bei der nächtlichen Zugunruhe gekäfigter Vögel. In Ornithologie als biologische Wissenschaft (Mayr, E. and Schüz, E., eds), pp. 269-283, Heidelberg

76 Itonaga, N. et al. (2010) Breeding dispersal directions in the white stork (Ciconia ciconia) are affected by spring migration routes. J. Ethol. 28, 393-397

77 Seeb, L.W. et al. (2007) Development of a standardized DNA database for Chinook salmon. Fisheries 32, 540-552 